\title{
Energy, Latency and Capacity Trade-offs in wireless multi-hop networks
}

\author{
Jean-Marie Gorce, Ruifeng Zhang, Katia Jaffrès-Runser, Claire Goursaud \\ University of Lyon, INRIA \\ INSA-Lyon, CITI, F-69621, France
}

\begin{abstract}
This paper concentrates on characterizing energy, latency and capacity trade-offs in multi-hop wireless ad-hoc networks. Therefore, a multiobjective framework is proposed to derive the Pareto-optimal set of solutions with respect to these three criteria. The work presented in this paper assumes a linear network where transmission powers and relay positions are optimization variables. We study the asymptotic state where the distance between source and destination is very high such that the number of hops tends to infinite. Two types of traffic are considered in the following. First, low rate traffic is analyzed by characterizing the multiobjective performance of a single packet transmission using an interference free multi-hop relaying strategy. Second, a continuous flow of packets from a unique source is considered. In the first case, we show an important theorem which states that all Pareto optimal solutions with respect to delay and energy metrics provide the same target SNR at the receiver side. In the second case, our analytical results highlight how the energy/delay Pareto front moves when considering a capacity constraint and the optimal re-use factor is derived.
\end{abstract}

\section{INTRODUCTION}

Evaluating the capacity of wireless ad hoc networks is an important issue that has triggered a comprehensive research effort under various conditions since the seminal work of Gupta and Kumar [1]. Several works provided ways for increasing the asymptotic capacity bound of $O(n \log (n))$ [1], [2] by for instance accounting for mobility [3] or introducing multi-user detection techniques [4]. Globally optimizing capacity for twodimensional networks where routing and resource allocation (i.e. frequency, time or power assignment) are performed concurrently is a very hard problem. Toumpis et al. [5] have proposed an interesting model to derive the capacity by properly scheduling transmissions and hence accounting for a temporal multiplexing directly in a 2 dimensional network model. While the model is appealing, the computation of optimal solutions for large networks is intractable due to the size of the search space.

A more tractable way to optimize multi-hop schemes is to consider a unique path in the network which can be assimilated to a linear network [6]-[10]. This leads to an optimization problem where the main variables are the transmission powers and locations of the relays. Common optimization objectives are energy minimization, latency minimization and capacity maximization.

Such a linear network model offers an important framework to derive a comprehensive analytical study of multihop transmission and has been investigated in the following works.
In [7], the authors have proposed to adapt the selection of the relays and their relative transmission powers to optimize power limited or bandwidth limited systems. Capacity and radiated energy are optimized but solutions using a high number of relays are favored since circuit energy is not accounted for. Further, no latency criterion is addressed. In [6] a circuit energy model is introduced for a direct single hop transmission. The same model is applied in [8] to evaluate multi-hop strategies but for fixed relay nodes. In their latest paper [10], the authors search for the multi-hop strategies maximizing the total throughput that also minimize the energy consumption. However, they do not account for interference in their model. They have extended their work to a spatial multiplexing context [9] but for a fixed set of relay nodes and the problem still concentrates on finding optimal powers.

The work presented in this paper pursues the same objectives and our contribution resides in the derivation of a multiobjective framework to optimize concurrently energy, delay and capacity. Results are here derived for a system where not only transmission powers are variable but also locations of relays can be adjusted. Analytical results rely on an asymptotic model where relays can be regularly positioned on a quasiinfinite line. In the first part of this work, we focus on the optimization of a single packet transmission when no spatial multiplexing is considered as in [10]. We derive the analytical Pareto front for the energy/latency trade-off, extending our previous work [11] which concentrated on computing these bounds only for specific modulations and coding techniques. In this paper, we provide a more general formulation where the achievable bound is characterized for any monotonically increasing capacity function. A specific derivation using the Shannon capacity illustrates the framework. We exhibit an important theorem which states that any Pareto optimal solution verifies the unique condition that the SNR at the receiver side is equal to an optimal SNR $\hat{\gamma}_{r}$. Our model is further extended to the case where a continuous flow is transmitted in the network. In this case, a channel re-use distance is introduced and interference is modeled as an additive Gaussian noise as in [7]. We first prove that the previous optimal SNR $\hat{\gamma}_{r}$ still holds as a bound but can be relaxed to target a specific capacity constraint. More specifically, the Pareto optimal set of solutions with respect to capacity maximization and energy/latency minimization is assessed by setting the received SNR in the range $\gamma_{r} \in\left[\hat{\gamma}_{r} ; \infty\right]$.

Section II introduces the model, assumptions and param- 
eters. The energy-per-bit and delay-per-bit objectives are defined. In Section III, the classical Shannon limit is revisited for a single hop transmission providing the latency/energy Pareto bound. In Section IV, the multi-hop transmission problem for a single packet transmission is addressed. The corresponding continuous flow problem is studied in Section V and Section VI concludes the paper.

\section{MOdel AND PARAMETERS}

A point to point transmission is being considered first. The transmission power density normalized with respect to the receiver noise power is denoted by $\gamma_{e}=\frac{P_{E} / W}{N_{0}}$, with $P_{E}$ the transmission power and $W$ the frequency bandwidth. On the receiver side, the normalized power density is the classical SNR denoted $\gamma_{r}$ and in the rest of the paper, all power and energy densities are normalized with respect to $N_{0}$.

The propagation environment is assumed homogenous, i.e. $\gamma_{r}=A(e, r) \cdot \gamma_{e}$ with:

$$
A(e, r)=A_{0} \cdot d_{e r}^{-n}
$$

where $n$ is the pathloss coefficient, $d_{e r}$ the emitter-receiver distance. The radio link capacity is:

$$
C=W \cdot c\left(\gamma_{r}\right)
$$

where the capacity per Hertz function $c(x)$ is supposed continuously derivable and monotonic. This function is noted $c_{g}(x)=\log (1+x)$ for the Gaussian channel.

For a given information quantity $Q_{i}$, the direct transmission delay $D_{i}$ is given by $D_{i}=Q_{i} / C$, which can be expressed as a delay-per-bit (latency):

$$
D_{b}=D_{i} / Q_{i}=C^{-1}
$$

The normalized radiated energy for transmitting $Q_{i}$ is:

$$
E_{i}=\gamma_{e} \cdot W \cdot D_{i}
$$

An additive component $E_{c}$ is introduced which stands for circuit energy and losses at both transmitter and receiver sides [6], [11]: $E_{t}=E_{i}+E_{c}$. This energy can also be normalized as an energy-per-bit quantity:

$$
E_{b}=E_{t} / Q_{i}
$$

\section{SINGLE HOP TRANSMISSION OPTIMIZATION}

In this section, Shannon's classical limit is revisited to express the energy/latency trade-off for transmitting $Q_{i}$ information bits. Capacity can be omitted because in a single hop transmission, capacity and latency are directly related to each other by (3). Thus, the following problem is considered: having a transmitter and a receiver at distance $d_{e r}$, what would be the optimal transmission power to concurrently reduce latency and energy consumption?

\section{A. Optimization for ideal transceivers}

Let's first consider a null circuit energy (i.e. $E_{c}=0$ ), thus:

$$
E_{b}=\gamma_{e} \cdot D_{i} \cdot W / Q_{i}
$$

Introducing (3) and (2), leads to:

$$
E_{b}=\gamma_{e} \cdot W / C=\frac{\gamma_{e}}{c\left(A(e, r) \cdot \gamma_{e}\right)}
$$

Besides, introducing (2) into (3) provides the delay per bit expression:

$$
D_{b}=\frac{1}{W \cdot c\left(A(e, r) \cdot \gamma_{e}\right)}
$$

Equations (7) and (8) give together the closed-form expression of the energy-latency Pareto front. The Pareto front represents the solutions that provide the best possible compromises between the considered performance metrics. In our case, it represents the optimal lower bound on the energy-latency trade-off. The compromise between both constraints is adjusted by setting the transmission power.

The inversion of (8) allows to express $\gamma_{e}$ as a function of a given delay constraint $D_{b}$ :

$$
E_{b}=\frac{W \cdot D_{b} \cdot c^{-1}\left(1 / W \cdot D_{b}\right)}{A(e, r)}
$$

In the Gaussian case, the Shannon's limit is obtained:

$$
E_{b}=\frac{W \cdot D_{b} \cdot\left(2^{1 / W \cdot D_{b}}-1\right)}{A(e, r)}
$$

This curve delimits the achievable region (above the curve) as represented in Fig.1 for $A(e, r)=1$. Any solution on this curve is Pareto optimal with respect to energy and latency.

\section{B. Effect of circuit energy}

For the case of non perfect transceivers, an additional circuit energy is consumed (10):

$$
E_{b}=\frac{W \cdot D_{b} \cdot c^{-1}\left(1 / W \cdot D_{b}\right)}{A(e, r)}+\frac{E_{c}}{Q_{i}}
$$

The circuit energy can be approximated [10] by:

$$
E_{c}=E_{c 0} \cdot Q_{i}+E_{\delta} \cdot D_{i} \cdot W
$$

where $E_{c 0}$ stands for the normalized digital circuit energyper-bit related to data processing and $E_{\delta} \cdot D_{i} \cdot W$ for the RF normalized per-bit-energy. Substituting (12) into (11) we get:

$$
\begin{aligned}
E_{b} & =\frac{W \cdot D_{b} \cdot c^{-1}\left(1 / W \cdot D_{b}\right)}{A(e, r)}+E_{c 0}+E_{\delta} \cdot D_{b} \cdot W \\
& =W \cdot D_{b} \cdot\left(\frac{c^{-1}\left(1 / W \cdot D_{b}\right)}{A(e, r)}+E_{\delta}\right)+E_{c 0}
\end{aligned}
$$

The constant circuit energy $E_{c 0}$ only shifts the curve along the energy axis and will be discarded in the rest of the paper. On the opposite, the component $E_{\delta}$ strongly affects the Pareto bound in the low power region, as illustrated in Fig.1 for $A(e, r)=1$. The plot of Fig.1 clearly shows that the RF energy-per-bit affects the right part of the curve and therefore reduces the achievable region. This figure exhibits the existence of a unique minimal energy solution, which limits the Pareto set to solutions with higher energy consumption. 


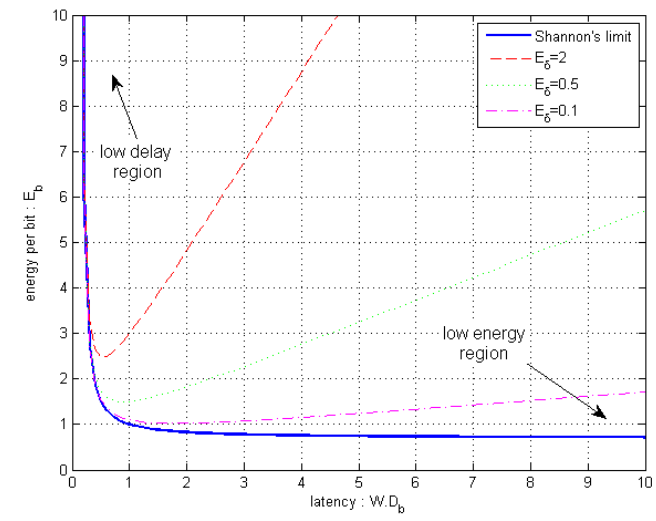

Fig. 1. Shannon's theoretical bound delimits the achievable region (above the curve) and represents the evaluation of the set of Pareto optimal solutions for energy/latency minimization. The RF energy consumption $E_{\delta}$ strongly affects the low energy region, and reveals an energy optimum point.

\section{LOW RATE MULTI-HOP OPTIMIZATION}

The previous section just revisited Shannon's well-known limit which is hereafter extended to the case of a multi-hop transmission. The problem is now to find what are the relay positions and their respective transmission powers achieving a Pareto optimal efficiency with respect to latency and energy criteria. This problem has been partially studied in [11] but only for specific transmission conditions where a link is characterized by its packet error rate and not its capacity. We here broaden the proposed formulation to a more general channel capacity model, allowing to derive an analytic expression of the optimal SNR. A similar problem has also been studied in [10] but the authors studied the case of fixed relays where only transmission powers can be optimized. They also consider the problem of energy minimization but without accounting for any latency constraint. Further, their optimal solutions are derived for specific scenarios while we concentrate on giving an asymptotic optimal solution.

\section{A. Theoretical Pareto front}

Let's consider that an information quantity $Q_{i}$ has to be transmitted over a very long distance and that we are free to choose the number and the location of the relays in order to optimize the transmission. The objective is to increase as much as possible the energy/delay feasible region, when compared to a direct transmission. In a homogeneous environment where the attenuation is just a function of the transmitter-receiver distance, the optimal solution corresponds to a regular distribution of the relays [7], [11], [12], providing all relays with the same power. Therefore, the optimization problem relies on finding the couple $\left(d_{h}, \gamma_{e}\right)$ where $d_{h}$ is the inter-node distance, and $\gamma_{e}$ the transmission power density. In this section, the transmission is interference free since the relays do not transmit simultaneously and a single packet is considered (see Fig.2).

Energy and latency constraints are now differentiated with respect to the distance, to obtain an asymptotic model, as proposed in [12]:

$$
\begin{aligned}
& \partial E_{b}\left(d_{h}, \gamma_{e}\right)=E_{b} / d_{h}=\frac{\gamma_{e}+E_{\delta}}{d_{h} \cdot c\left(A_{0} \cdot d_{h}^{-n} \cdot \gamma_{e}\right)} \\
& \partial D_{b}\left(d_{h}, \gamma_{e}\right)=D_{b} / d_{h}=\frac{1}{d_{h} \cdot W \cdot c\left(A_{0} \cdot d_{h}^{-n} \cdot \gamma_{e}\right)}
\end{aligned}
$$

These parametric equations represent respectively the energy and delay needed to transmit one bit over one meter referred to as the per-bit-per-meter energy, and the per-meter bit latency, as a function of $\left(d_{h}, \gamma_{e}\right)$. The total latency and per-bit energy are derived after multiplying with the total distance $d_{t o t}$.

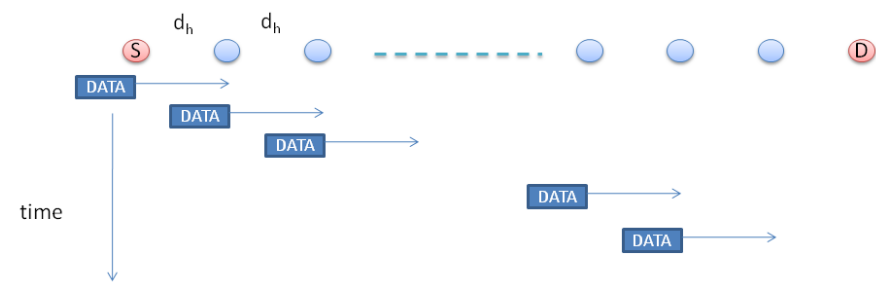

Fig. 2. The first scenario concerns the transmission of a single packet along a line, with a regular multi-hop approach

The achievable region bound can be assessed from (14). The problem relies on finding the couples $\left(d_{h}, \gamma_{e}\right)$ that are Pareto optimal. We now change (14) thanks to the attenuation model given in (1) to work with $\left(\gamma_{r}, \gamma_{e}\right)$ instead of $\left(d_{h}, \gamma_{e}\right)$. This simple variable change allows to draw very interesting conclusions as stated now.

(14) can be rewritten as such:

$$
\begin{aligned}
\delta E_{b}\left(\gamma_{e}, \gamma_{r}\right) & =\frac{\gamma_{e}+E_{\delta}}{\left(A_{0} \cdot \gamma_{e}\right)^{1 / n}} \cdot \frac{\gamma_{r}^{1 / n}}{c\left(\gamma_{r}\right)} \\
\delta D_{b}\left(\gamma_{e}, \gamma_{r}\right) & =\frac{1}{W \cdot\left(A_{0} \cdot \gamma_{e}\right)^{1 / n}} \cdot \frac{\gamma_{r}^{1 / n}}{c\left(\gamma_{r}\right)}
\end{aligned}
$$

These equations turn out to be separable with respect to $\gamma_{e}$ and $\gamma_{r}$, i.e. $\delta E_{b}\left(\gamma_{e}, \gamma_{r}\right)=f_{E}\left(\gamma_{e}\right) \cdot g_{E}\left(\gamma_{r}\right)$ and $\delta D_{b}\left(\gamma_{e}, \gamma_{r}\right)=$ $f_{D}\left(\gamma_{e}\right) \cdot g_{D}\left(\gamma_{r}\right)$. Further, highlighting that $g_{E}$ and $g_{R}$ are both equal to $g(\gamma)=\frac{\gamma^{1 / n}}{c(\gamma)}$, leads to the following theorem:

Theorem 1: In a homogeneous environment characterized by a power-law attenuation (1) and a capacity function $c\left(\gamma_{r}\right)$ monotonically increasing, a regular linear network is Pareto optimal with respect to energy and latency if and only if :

$$
\begin{gathered}
\hat{\gamma}_{r}=\arg \min g(\gamma) \\
\hat{\gamma}_{e} \geq \frac{E_{\delta}}{n-1}
\end{gathered}
$$

If $c\left(\gamma_{r}\right) / c^{\prime}\left(\gamma_{r}\right)$ is monotonous, the solution of (16) is unique.

Proof: First, the cost functions are separable with respect to $\gamma_{r}$ and $\gamma_{e}$. Second, $\gamma_{r}$ and $\gamma_{e}$ are independent because the inter-node distance is not fixed. Since $g_{E}()$ and $g_{R}()$ are equal, the joint minimization of (15) with respect to $\gamma_{r}$ is achieved only when $g\left(\gamma_{r}\right)$ is minimal, which prooves (16).

Concerning (17), the achievable bound varies with different parameters (e.g. $\left.E_{\delta}, n, \ldots\right)$ but always looks like the curve in 
Fig.1. Thus, due to circuit energy, a minimal energy point exists. Therefore, the Pareto optimal set corresponds to the part of the achievable bound that is on the left of the minima, since on the right, both energy and latency increase. The minima correspond to $\hat{\gamma}_{e}=\arg \min f_{E}\left(\gamma_{e}\right)$.

This result asserts that any solution on the Pareto front is obtained by jointly tuning $\gamma_{e}$ and $d_{h}$ to maintain $\hat{\gamma}_{r}$ constant. Therefore, the inter-node distance $d_{h}$ can be used as a tunable parameter to fit with transmitter and receiver SNR constraints. Energy and latency are then balanced thanks to $\gamma_{e}$. A corollary of the previous theorem follows:

Theorem 2: In a homogeneous environment characterized by (1) and a capacity function $c\left(\gamma_{r}\right)$ monotonously increasing, a regular linear relaying network is Pareto optimal with respect to energy and delay if and only if $\gamma_{e}$ and $d_{h}$ are related by:

$$
d_{h}^{n}=\frac{A_{0}}{\hat{\gamma}_{r}} \cdot \gamma_{e} ; \forall \gamma_{e} \geq \frac{E_{\delta}}{n-1}
$$

Proof: Since $\gamma_{r}$ is constrained by theorem 1, theorem 2 follows from (1).

\section{B. Energy minimization}

The minimal per-bit-per-meter energy corresponding to $\hat{\gamma}_{e}$ is given by :

$$
\partial E_{b}=\frac{n \cdot g_{0}}{(n-1)^{(n-1) / n}} \cdot \frac{E_{\delta}^{(n-1) / n}}{A_{0}^{1 / n}}
$$

where $g_{0}=g\left(\hat{\gamma}_{r}\right)$. One can highlight that the minimal per-bit energy depends only on the pathloss coefficient and the circuit energy. The corresponding per-meter bit latency is :

$$
\partial D_{b}=\frac{(n-1)^{1 / n} \cdot g_{0}}{W \cdot\left(A_{0}\right)^{1 / n}} \cdot E_{\delta}^{-1 / n}
$$

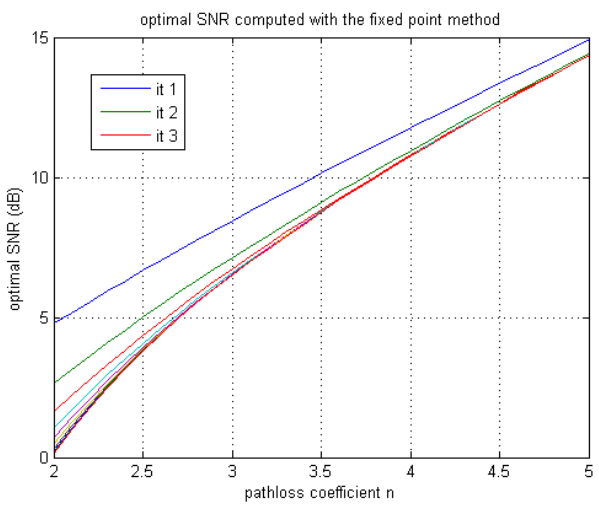

Fig. 3. Optimal SNR $\hat{\gamma}_{r}$ as a function of the pathloss coefficient, and computed with the fixed point method which converges in a few iterations.

\section{Application to Gaussian channels}

Results can be evaluated for an ideal Gaussian channel.Solving (16) leads to:

$$
n \gamma_{r}=\left(1+\gamma_{r}\right) \cdot \log \left(1+\gamma_{r}\right)
$$

The solution exists, is unique and is only a function of the pathloss coefficient $n$ as shown in Fig.3. For instance, $n=3$, $\hat{\gamma}_{r}=6.5 d B$ corresponds to $g_{0}=g\left(\hat{\gamma}_{r}\right)=0.67$. The minimal energy is $\partial E_{b}=1.266 \cdot E_{\delta}^{2 / 3} / A_{0}^{1 / 3}$. The achievable region is depicted in Fig.4.

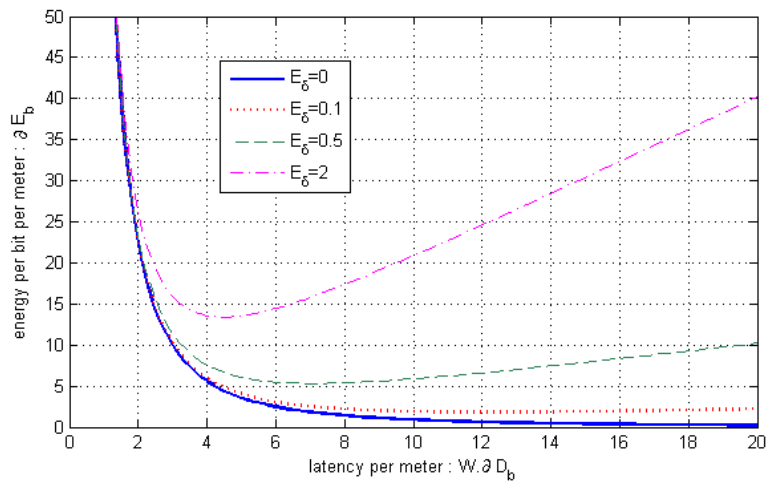

Fig. 4. The Shannon's based frontier for the achievable region is represented for $A_{0}=1, n=3$ and for different circuit energy values

\section{HIGH RATE MULTI-HOP OPTIMIZATION}

In the previous scenario, a relay transmits only one after the other and thus the capacity tends to 0 for an infinite linear network. Now, a continuous data flow transmission (see Fig.5) is studied thanks to a spatial re-use policy. We here aim at getting a picture of the compromises existing between the perbit-per-meter energy, the per-meter bit latency and the capacity of the system.

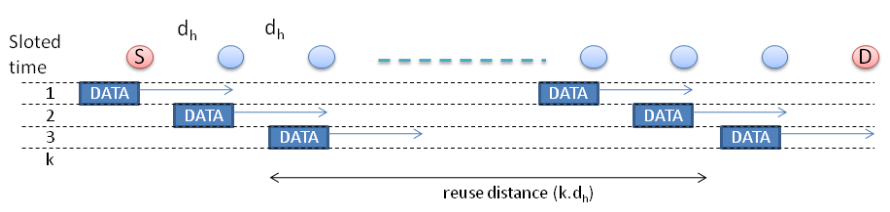

Fig. 5. The second scenario corresponds to a transmission of a continuous flow along an infinite linear network using a regular spatial multiplexing

\section{A. Problem formulation}

Without loss of generality, let's consider a TDMA multiplexing scheme using $k$ slots. The new optimization problem relies on finding the set of triplets $\left(k, \gamma_{e}\right.$ and $\left.\gamma_{r}\right)$ that achieves a Pareto optimal solution with respect to energy, latency and capacity.

For the link capacity, SINR $\Gamma_{I}$ now replaces $\gamma_{r}$ :

$$
\Gamma_{I}=\frac{\gamma_{r}}{1+I(k) \cdot \gamma_{r}}
$$

where $I(k)$ is the relative received interference (see Fig.6) given by [7]: $I(k)=\sum_{i=-\infty}^{\infty} \beta_{i}(k)$. From simple geometric rules, one have:

$$
I(k)=\sum_{i=1}^{\infty}\left((i \cdot k-1)^{-n}+(i \cdot k+1)^{-n}\right)
$$


The first and second terms in the sum correspond respectively to the forward and backward interference. A coarse approximation of (23) will be used for the sake of simplicity by considering only the first $k$ interfering signal, i.e. $I(k)>$ $(k-1)^{-n}$. The link capacity as a function of $\gamma_{r}$ is obtained by putting $(22)$ in $c(x)$ :

$$
c^{(k)}\left(\gamma_{r}\right)=c\left(\frac{\gamma_{r}}{1+I(k) \cdot \gamma_{r}}\right)
$$

The system of equations (15) of Section IV still holds for the energy/latency trade-off and when there is no capacity constraint, the Pareto bound is achieved when $\hat{\gamma}_{r}^{(k)}=$ $\arg \min g^{(k)}\left(\gamma_{r}\right)$.

The multi-hop capacity of the system is:

$$
C_{m h}\left(\gamma_{r}, k\right)=\frac{c^{(k)}\left(\gamma_{r}\right)}{k}
$$

The maximal capacity is obtained for an infinite power:

$$
C_{\infty}(k)=\lim _{\gamma_{r} \rightarrow \infty} C_{m h}\left(\gamma_{r}, k\right)=c\left(I(k)^{-1}\right)
$$

which can be bounded by $C_{\infty}(k)<\frac{c\left(1+(k-1)^{n}\right)}{k}$, thanks to the first order approximation of $I(k)$.

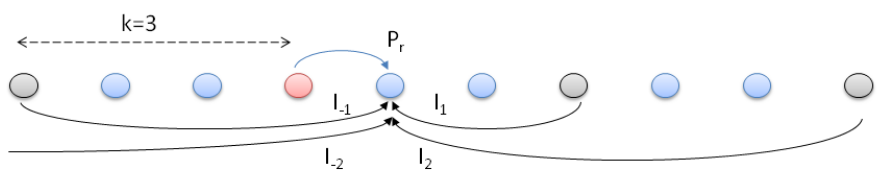

Fig. 6. The interference strength relies on the re-use factor in a periodic structure.

\section{B. Optimization with a constant re-use factor}

Let's now study how to trade between capacity, latency and energy for a specific re-use factor $k$.

Theorem 3: In a homogeneous environment characterized by (1) and a capacity function $c\left(\gamma_{r}\right)$, the Pareto optimal solutions with respect to capacity, energy and latency for a given re-use factor $k>1$ are defined by the following constraints :

- the received SNR verifies: $\hat{\gamma}_{r}^{(k)}<\gamma_{r}<\infty$, with $\hat{\gamma}_{r}^{(k)}=\arg \min g^{(k)}(x)=x^{1 / n} / c^{(k)}(x)$

- the transmitted power density $\gamma_{e}$ verifies : $\gamma_{e} \geq E_{\delta} /(n-$ 1).

Proof: First, note that capacity only depends on the received SINR $\Gamma_{I}$ and is not affected by the couple $\left(d_{h}, \gamma_{e}\right)$ if (18) is fulfilled. Obviously, the higher $\gamma_{r}$, the higher $\Gamma_{I}$ and the higher the capacity. On the contrary, $\gamma_{r}$ should be kept close to $\hat{\gamma}_{r}$ to cope with energy/latency constraints. Thus, $\gamma_{r}$ allows to balance between capacity and energy/latency. For $\gamma_{r}<\hat{\gamma}_{r}$ the solution is not Pareto optimal since capacity decreases while energy and latency increase.

Second, $\left(\gamma_{e}, d_{h}\right)$ values do not affect the capacity and can be used to balance latency and energy only. Note that the receive SINR $\Gamma_{I}$ is bounded by $\hat{\gamma}_{r} /\left(1+(k-1)^{-n} \cdot \hat{\gamma}_{r}\right)<\Gamma_{I}<$ $(k-1)^{n}$
Note that the optimization problem was shown separable with respect to $\gamma_{e}$ and $\gamma_{r}$. Besides, without latency constraint, the optimal transmitted power and inter-node distance are respectively $\tilde{\gamma}_{e}=\frac{E_{\delta}}{n-1}$ and $\hat{d}_{h}=\left(\frac{A_{0} \cdot \tilde{\gamma}_{e}}{\tilde{\gamma}_{r}}\right)^{1 / n}$ Thus, all energy/capacity optimal solutions are achieved with the same transmission power which means that adapting the node density instead of the transmission powers is the optimal strategy.

The two extrem cases corresponding to the minimal energy solution and to the maximal capacity are:

a) minimal energy solution: The minimal per-bit-permeter energy is obtained for $\gamma_{r}=\hat{\gamma}_{r}^{(k)}$ and is proportional to $g_{0}^{(k)}$ :

$$
\partial E_{b}=\frac{n \cdot g_{0}^{(k)}}{(n-1)^{(n-1) / n}} \cdot \frac{E_{\delta}^{(n-1) / n}}{A_{0}^{1 / n}}
$$

The corresponding capacity is $C_{m h}\left(\hat{\gamma}_{r}^{(k)}, k\right)$.

b) maximal capacity solution: The maximal capacity $C_{\infty}$ (as defined in (26)) is achieved for an infinite SINR, and corresponds to an infinite per-meter-per-bit energy.

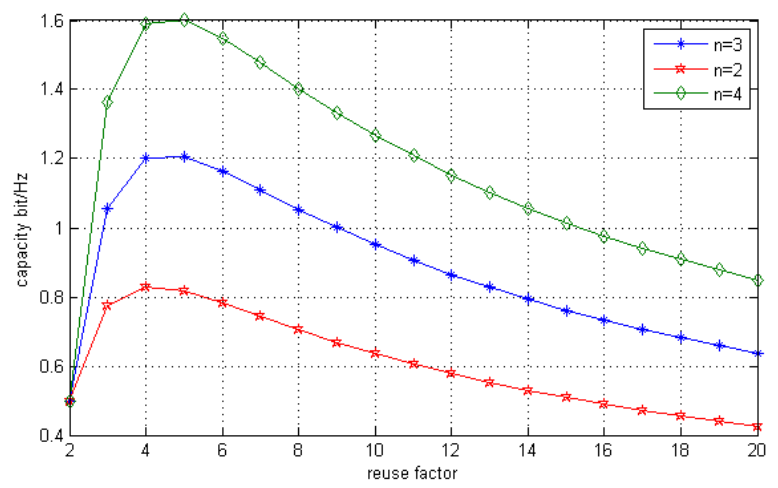

Fig. 7. Maximal capacity in bit/Hz in a Gaussian channel as a function of the attenuation factor $n$ and the re-use factor $k$

\section{Re-use factor optimization for Gaussian channels}

The optimal re-use factor is the one corresponding to the maximal capacity under a given energy/latency constraint. We first consider the capacity bound $C_{\infty}(k)<\frac{c\left(1+(k-1)^{n}\right)}{k}$ which is a ratio of two mononitically increasing functions and the optimal value depends on the capacity function ( see Fig. 7 ). This plot represents the maximal capacity that can be achieved with a non-limited transmission power. In a Gaussian channel, the optimal solution is $k \in\{4,5\}$. Now the capacity objective has to be balanced with the energy/latency objectives.

From our previous analysis, the minimal SINR that allows to reach the energy/latency Pareto front is known but leads to a sub-optimal capacity $C_{m h}\left(\hat{\gamma}_{r}^{(k)}, k\right)$. This trade-off is illustrated on Fig.8 for a pathloss exponent $n=3$. The achievable region bound between energy/latency and capacity is represented through $g\left(\gamma_{r}\right)$ and $C_{m h}\left(\gamma_{r}, k\right)$. The highest capacity is achieved for $k=5$ but for a high SNR. We see on this figure how increasing $k$ allows to decrease $g_{0}^{(k)}$ at the price of a reduced capacity. From $k=2$ up to $k=5$, 
the achievable region increases significantly. For higher $k$ values, the achievable region shifts to the left and thus favors energy/latency upon capacity. As a conclusion, we show that for $n=3$, the smaller re-use factor is $k=5$. When the energy constraint is very strong, $k$ can be increased up to 10, but leads to a capacity loss of about $60 \%$. Note that the minimal perbit-per-meter energy is equal to the one computed with no multiplexing.

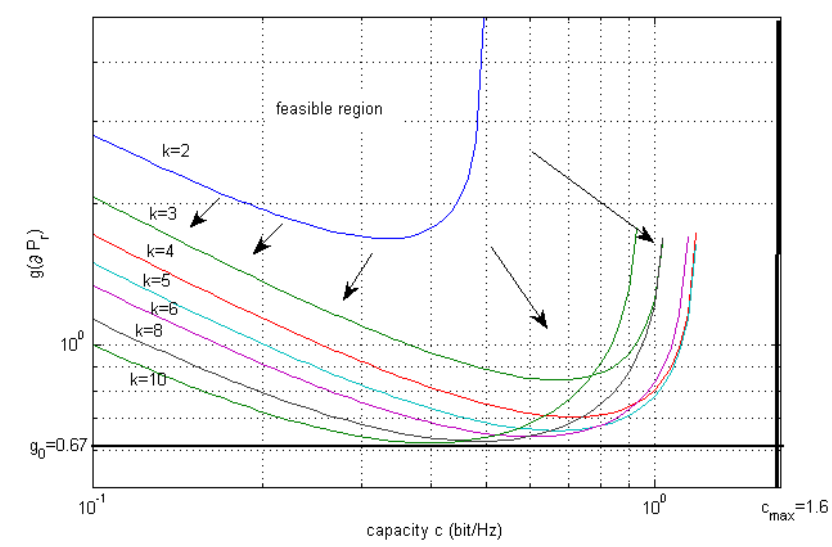

Fig. 8. The achievable region in terms of capacity vs energy/latency is given for different re-use factors. The highest capacity is achieved for $k=5$.

\section{CONCLUSION AND DISCUSSIONS}

In this paper, we derived a general approach to analyze how energy, latency and capacity are linked together. We have first evaluated the energy/latency trade-off for a single packet transmission over a regular linear network and derived optimal transmission power and inter-node distance accordingly.

From a practical point of view, we have proved that all optimal solutions with respect to latency and energy are achieved for a constant received SNR $\hat{\gamma}_{r}$ that is related to the capacity function and the path-loss coefficient only. On the other side, the optimal transmission power is obtained as a function of the circuit energy. The inter-node distance is then chosen to comply with the transmitting and receiving SNR constraints.

We have also extended this framework for a continuous flow by introducing a re-use factor. We have obtained the optimal re-use factor and derived the Pareto bound with respect to the three constraints of energy, latency and capacity. We proved that Pareto optimal solutions with respect to energy and capacity are obtained for a constant transmission power that is related to the circuit energy and the pathloss coefficient only. Then, the received SNR and the re-use factor $k$ are set to fulfill a target capacity constraint, and the inter-nodfe distance is again used to comply with both sides constraints. The knowledge of the optimal inter-node distance can be exploited in real deployments, to optimize the nodes' density and thus to ensure a good probability of having some nodes at a right distance, or to define a good wake-up policy for a dense network.
These results claim that an optimal routing approach in a wireless sensor network, whatever the optimization constraints, should be rather based on selecting the relays located at the optimal inter-node distance, instead of changing the transmission powers if the environement is homogeneous. Further, it seems that controlling the received SINR to be constant is probably very efficient to comply with inhomogeneous environments.

These results are very complementary to those obtained previously in [7] and offer a very comprehensive framework to represent the performance of relaying approaches. Future works could propose to evaluate other relaying strategies within the same multi-objective framework such as:

- Interference mitigation or avoidance techniques can be evaluated by removing the forward interference in 23 [7].

- Amplify-and-Forward could be introduced in the model to allow several nodes on the line to cooperate.

- virtual MIMO techniques could be considered.

\section{REFERENCES}

[1] P. Gupta and P. Kumar, "The capacity of wireless networks," IEEE transactions on information Theory, vol. 46, pp. 388-404, march 2000.

[2] Z. Wang, H. Sadjadpour, and J. Garcia-Luna-Aceves, "A unifying perspective on the capacity of wireless ad hoc networks," in INFOCOM 2008. The 27th Conference on Computer Communications. IEEE, april 2008, pp. $211-215$.

[3] M. Grossglauser and D. N. C. Tse, "Mobility increases the capacity of ad hoc wireless networks," IEEE/ACM Trans. Netw., vol. 10, no. 4, pp. 477-486, 2002

[4] C. Comaniciu and H. Poor, "On the capacity of mobile ad hoc networks with delay constraints," Wireless Communications, IEEE Transactions on, vol. 5, no. 8, pp. $2061-2071$, aug. 2006.

[5] S. Toumpis and A. Goldsmith, "Capacity regions for wireless ad hoc networks," Wireless Communications, IEEE Transactions on, vol. 2, no. 4, pp. $736-748$, july 2003 .

[6] S. Cui, A. Goldsmith, and A. Bahai, "Energy-constrained modulation optimization," Wireless Communications, IEEE Transactions on, vol. 4, no. 5, pp. 2349 - 2360, sept. 2005.

[7] M. Sikora, J. N. Laneman, M. Haenggi, D. J. Costello, Jr., and T. E. Fuja, "Bandwidth- and power-efficient routing in linear wireless networks," IEEE/ACM Trans. Netw., vol. 14, no. SI, pp. 2624-2633, 2006.

[8] S. Cui, R. Madan, A. Goldsmith, and S. Lall, "Cross-layer energy and delay optimization in small-scale sensor networks," Wireless Communications, IEEE Transactions on, vol. 6, no. 10, pp. 3688 -3699, october 2007.

[9] C. Bae and W. Stark, "Energy-bandwidth tradeoff with spatial reuse in wireless multi-hop networks," in Military Communications Conference, 2008. MILCOM 2008. IEEE, nov. 2008, pp. $1-7$.

[10] C. Bae and W. E. Stark, "End-to-end energy-bandwidth tradeoff in multihop wireless networks," IEEE Trans. Inf. Theor, vol. 55, no. 9, pp. 4051-4066, 2009.

[11] R. Zhang and J. M. Gorce, "Optimal transmission range for minimum energy consumption in wireless sensor networks," in Wireless Communications and Networking Conference, 2008. WCNC 2008. IEEE, Las Vegas, NV, 2008, pp. 757-762.

[12] R. Zhang, "Analysis of energy-delay performance in multi-hop wireless sensor networks," Ph.D. dissertation, INSA de Lyon, 2009. 